\title{
Quantum Distribution Functions for Dusty Plasma in Saturn's Rings by Using Curvilinear Coordinates
}

\author{
Nagat Abd El Rahman Hussein ${ }^{1}$, Abd El-Rahim Hassan Ahmed ${ }^{2}$, Esraa Gamal Sayed ${ }^{*}$ \\ ${ }^{1}$ Mathematics Department, Faculty of Science, Assiut University, Assiut, Egypt \\ ${ }^{2}$ Mathematics Department, Faculty of Science, Al-Azhar University, Assiut, Egypt \\ Email: *aragamal@yahoo.com
}

How to cite this paper: Hussein, N.A. El R., Ahmed, A.El-R.H. and Sayed, E.G. (2019) Quantum Distribution Functions for Dusty Plasma in Saturn's Rings by Using Curvilinear Coordinates. International Journal of Astronomy and Astrophysics, 9, 115-132.

https://doi.org/10.4236/ijaa.2019.92009

Received: February 13, 2019

Accepted: May 26, 2019

Published: May 29, 2019

Copyright $\odot 2019$ by author(s) and Scientific Research Publishing Inc. This work is licensed under the Creative Commons Attribution International License (CC BY 4.0).

http://creativecommons.org/licenses/by/4.0/

\begin{abstract}
This study aims to estimate the quantum Bogoliubov-Born-Green-KirkwoodYvon (BBGKY) hierarchy in curvilinear coordinates. We used the results to calculate the quantum binary and triplet distribution functions in curvilinear coordinates. The analytical form of the quantum distribution functions was obtained for dusty plasma in Saturn's rings model. We use particles-in-cell (PIC) simulations to find a visualization of dusty three-component plasma phase space in curvilinear coordinates. Our results were compared with others.
\end{abstract}

\section{Keywords}

Dusty Plasma, Binary and Triplet Distribution Functions, Three Component Plasma, BBGKY Hierarchy

\section{Introduction}

The quantum distribution functions are of great interest for understanding the properties of dusty plasma. In statistical mechanics, we can get the thermodynamic functions such as the internal energy, the osmotic pressure, and the excess free energy by using the distribution functions for the plasma particles. The importance of quantum distribution function in statistical physics is to give the particles number density in the phase space at time $t$.

Many authors have calculated the quantum distribution functions. Hussein and Hassan [1] have calculated the quantum binary distribution of high temperature plasma. Kraeft et al. [2] used effective potentials to calculate the binary distribution function. Bogoliubov and Kraeft et al. [3] [4] defined a set of equations describing the dynamics of a system of a large number of interacting par- 
ticles by using the quantum BBGKY hierarchy. Kaniadakis [5] showed that the classical BBGKY hierarchical equation, for the n-particle reduced distribution function. Fesciyan [6] calculated the quantum time independent BBGKY hierarchy of equations at low densities. Hussein et al. [7] used the BBGKY hierarchy for calculating the binary and triplet distribution functions for one- and two-component Plasmas in terms of Green's function technique. A quantum mechanical calculation of the radial distribution function for a Plasma was given by [8]. Also, many others have studied the binary and triplet distribution functions [9]-[19]. Many researchers have been interested in identifying the optimal form of the classical distribution functions [16]. Hansen [17] calculated the distribution functions for one component plasma in the classical form and quantum corrections.

The calculation of the distribution functions in general form by using curvilinear coordinates makes it easier for the researchers to find the form of the distribution function in the spherical or cylindrical coordinates or any type of coordinates that facilitates the study of the physical problem. Błaszak and Domanski [20] calculated the canonical quantization of classical mechanics in curvilinear coordinates. Kjaergaard and Mortensen in 1990 [21] made a simple derivation of the quantum mechanical Hamiltonian in curvilinear coordinates. In 2012 a generalized, curvilinear-coordinate formulation of Poisson's equations to solve for the electrostatic fields in plasma was given by Fichtl et al. [22].

Dusty Plasmas plays an important role in experimental physics and in many astrophysical situations [23]. To understand many space and astrophysical phenomena, and many industrial and physical applications, the study of plasmas containing heavy dust particles is very important [24]. It differs from ordinary plasma in the presence of dust particles along with a number of positive and negative charges moving at a high speed if compared with the speed of dust [25]. Wang and Zhang [26] employed the quantum hydrodynamic model to study the solution and chaotic structures of dust ion-acoustic waves in quantum dusty plasmas consisting of electrons, ions and charged dust particles.

Dusty plasma was originally important in the field of astrophysics. Examples of astronomical dusty plasmas include planetary ring systems (rings of Saturn). The rings of Saturn are the most important models in space dusty plasma study [27]. The rings of Saturn are made of billions of particles; these particles mostly range from tiny, dust-sized icy grains to giant chunks. They are made of small chunks of ice and rock coated with another material such as dust. A few particles are as large as mountains.

Saturn's ring system extends up to 175,000 miles (282,000 kilometres) from the planet, yet the vertical height is typically about 30 feet (10 meters) in the main rings [28]. Named alphabetically in the order they were discovered, the rings are relatively close to each other, with the exception of a gap measuring 2920 miles (4700 kilometres) wide called the Cassini Division that separates Rings $\mathrm{A}$ and $\mathrm{B}$. The main rings are A, B and C. Rings D, E, F and G are fainter and more recently discovered; each ring orbits at a different speed around the 
planet [29]. But if we ask a question why plasma molecules move in these rings in circular paths and what is the nature of their components. And how to find the distribution functions of their particles. Does the use of curvilinear coordinates make it easier to find and deal with the functions of predisposition? In this study, we attempted to find the form of the distribution functions of the molecules of planetary rings. A model was found that simulates its shape. How fast the particles of planetary rings and whether it is necessary to use Einstein patches for high speeds. In this research, we tried to answer some of these questions.

Particle-in-cell (PIC) simulations are a useful tool in modelling plasma. The electron velocity distribution function and the plasma potential are found by particle-in-cell (PIC) simulations [30]. Reinmüller in 1998 [31] determined the plasma potential from PIC simulations. We use particle-in-cell (PIC) simulations to find a visualization of dusty three component plasma quantum phase space in curvilinear coordinates.

This work is aimed to calculate the quantum binary and triplet distribution functions of a dusty plasma in curvilinear coordinates. In geometry, curvilinear coordinates are a coordinate system for Euclidean space in which the coordinate lines may be curved. Commonly used curvilinear coordinate systems include rectangular, spherical, and cylindrical coordinate systems. The calculation is based on the Bogoliubov-Born-Green-Kirkwood-Yvon (BBGKY) hierarchy [3].

The cluster expansion method consists of writing the binary distribution function as a power series in the density. The coefficients of different powers of the density involve integrals of the order of that power. These coefficients are then expressed as a sum of a product of integrals. The power series of the binary distribution function in the density convergence badly at high densities, many attempts have been made to overcome such difficulty.

\section{The Basic Equations and Hierarchy}

Consider 3 Dimensions space with coordinates $X=\left(x_{1}, x_{2}, x_{3}\right)$. A point $\mathrm{p}$ in $3 \mathrm{~d}$ space can be defined using Cartesian coordinates or it can also be defined by its curvilinear coordinates $\xi=\left(\xi_{1}, \xi_{2}, \xi_{3}\right)$. The relation between the coordinates is then given by the invertible transformation functions:

$$
\begin{aligned}
\xi_{s} & =\xi_{s}\left(x_{1}, x_{2}, x_{3}\right), s=1,2,3 \\
x_{i} & =x_{i}\left(\xi_{1}, \xi_{2}, \xi_{3}\right), i=1,2,3
\end{aligned}
$$

The surfaces $\xi_{1}=$ constant, $\xi_{2}=$ constant, $\xi_{3}=$ constant are called the coordinate surfaces. The coordinate axes are determined by the tangents to the coordinate curves at the intersection of three surfaces. They are not in general fixed directions in space, which happens to be the case for simple Cartesian coordinates, and thus there is generally no natural global basis for curvilinear coordinates. The momentum operator in quantum mechanics is the gradiant operator $p=(\hbar / i) \nabla$. By defining the Jacobi matrix as: 


$$
j_{\alpha \beta}=\frac{\partial x^{\alpha}}{\partial \xi^{\beta}}, \alpha, \beta=1,2,3 .
$$

The Jacobian of the transformation is the determinant of the Jacobi matrix

$$
J(\xi)=\operatorname{det}\left[j_{\alpha \beta}\right]
$$

Define the natural basis vectors:

$$
\begin{gathered}
h_{i}=\frac{\partial r}{\partial \xi_{\alpha}}, \alpha=1,2,3 \\
r=\left(x_{1}, x_{2}, x_{3}\right)
\end{gathered}
$$

The reduced $s$-particle density operators defined by Bogoliubov [3] in the following form

$$
F_{s}\left(\xi_{1}, P_{\xi_{1}}, \xi_{2}, P_{\xi_{2}}, \cdots, \xi_{N}, P_{\xi_{N}}\right)=V^{s} \operatorname{Tr}_{\xi_{s+1} \cdots \xi_{N}} Q^{-1} \exp \left(-\beta H_{N}\right) ;
$$

where $H_{N}$ is the Hamiltonian of our system given by

$$
H_{N}=\frac{-\hbar^{2}}{2 m} \nabla^{2}+V(\xi)
$$

where $V$ is the potential of the system and $Q$ is the configuration integral is given by

$$
Q=T r_{\xi_{s+1} \cdots \xi_{N}} \mathrm{e}^{-\beta H_{N}},
$$

The solution of $\mathrm{N}$-particle schrödinger (time-dependent) equation with this Hamiltonian are given by $\left|\Psi^{(1)}\right\rangle \ldots\left|\Psi^{(M)}\right\rangle$ and from a complete orthonormal basis

$$
\begin{gathered}
\left\langle\Psi^{(1)}\left|p_{n}\right| \Psi^{(2)}\right\rangle=\frac{\hbar}{i} \int \mathrm{d} \xi_{1} \int \mathrm{d} \xi_{2} \int \mathrm{d} \xi_{3} \sqrt{J} \Psi_{1}^{*} \frac{\partial \sqrt{J} \Psi^{(2)}}{\partial \xi_{n}} \\
\sum_{k=1}^{M}\left|\Psi^{(k)}\right\rangle\left\langle\Psi^{(k)}\right|=1
\end{gathered}
$$

Define the $\mathrm{N}$-particle density operator

$$
\hat{\rho}=\sum_{k=1}^{M} w_{k}\left|\Psi^{(k)}\right\rangle\left\langle\Psi^{(k)}\right|
$$

where $w_{k}$ are positive real probabilities

$$
\begin{aligned}
& \sum_{k=1}^{M} w_{k}=1 \\
& 0 \leq w_{k} \leq 1
\end{aligned}
$$

The density operator $\hat{\rho}$ follow the Von Neumann equation

$$
i \hbar \frac{\partial}{\partial t} \hat{\rho}-[\hat{H}, \hat{\rho}]=0
$$

In order to derive the quantum BBGKY-hierarchy, we introduce the reduced $s$-particle density operator as

$$
\hat{F}_{1 \ldots s}=C_{s}^{N} \operatorname{Tr}_{s+1 \cdots N} \hat{\rho}
$$


and $\operatorname{Tr}_{1 \ldots s} \hat{F}_{1 \ldots s}=C_{s}^{N}=\frac{N !}{(N-s) !}$.

The equation of motion for the reduced density operator obeys directly the Von Neuman equation. Now by substituting from Equation (12) into Equation (11) we get

$$
i \hbar \frac{\partial}{\partial t} \hat{F}_{1 \ldots s}-\left[\hat{H}_{1 \ldots s}, \hat{F}_{1 \ldots s}\right]=T r_{s+1} \sum_{i=1}^{s}\left[\hat{v}_{i, s+1}, \hat{F}_{1 \cdots s+1}\right]
$$

where $\hat{v}_{i, j}$ is the potential between particles $i, j$ and $\hat{H}_{1 \ldots s}$ is the s-particle Hamiltonian operator. The above equation constitutes the quantum generalization of the (BBGKY) hierarchy.

\section{The Binary Distribution Function}

We assume that the momentum of the electron lies between $\xi_{1}$ and $\xi_{1}+\mathrm{d} \xi_{1}$ is $P_{\xi_{1}}$. Also the momentum of the positron lies between $\xi_{2}$ and $\xi_{2}+\mathrm{d} \xi_{2}$ is $P_{\xi_{2}}$ and the momentum of the dust (ion) lies between $\xi_{3}$ and $\xi_{3}+\mathrm{d} \xi_{3}$ is $P_{\xi_{3}}$. In this section, we shall find the binary distribution function of a quantum dusty in curvilinear coordinates. Firstly, define the quantum $\mathrm{N}$ particle distrbution function as follows:

$$
F_{N}^{q u}\left(\xi_{N}, P_{\xi_{N}}\right)=\frac{N ! \exp \left[-\beta \sum_{i=1}^{N} \frac{P_{\xi_{i}}^{2}}{2 m}-\beta U\left(\xi_{N}\right)\right]}{\int \mathrm{d} P_{\xi_{N}} \exp \left[-\beta \sum_{i=1}^{N} \frac{P_{\xi_{i}}^{2}}{2 m}\right] \int \mathrm{d} \xi_{N} \exp \left[-\beta U\left(\xi_{N}\right)\right]}
$$

The one particle distribution function is obtained by reducing $F_{N}^{q u}\left(\xi_{N}, P_{\xi_{N}}\right)$ by integrating over $N-1$ positions and momenta then

$$
F_{1}^{q u}\left(\xi_{1}, P_{\xi_{1}}\right)=\frac{1}{(N-1) !} \iint \mathrm{d} \xi_{N} \mathrm{~d} P_{\xi_{N}} F_{N}^{q u}\left(\xi_{N}, P_{\xi_{N}}\right)
$$

If there are no external fields

$$
F_{1}^{q u}\left(\xi_{1}, P_{\xi_{1}}\right)=\alpha \exp \left[\frac{-\beta}{2 m} P_{\xi_{1}}^{2}\right]
$$

where the value of $\alpha$ can be found by normalization:

$$
\iint \mathrm{d} \xi_{1} \mathrm{~d} P_{\xi_{1}} F_{1}^{q u}\left(\xi_{1}, P_{\xi_{1}}\right)=N
$$

By substituting from Equation (16) into Equation (17) we get

$$
\alpha^{-1}=\frac{V}{N}\left(\frac{2 \pi m}{\beta}\right)^{3 / 2}
$$

Then the one particle distribution function is given by

$$
F_{1}^{q u}\left(\xi_{1}, P_{\xi_{1}}\right)=\frac{N}{V}\left(\frac{\beta}{2 \pi m}\right)^{3 / 2} \exp \left[\frac{-\beta}{2 m} P_{\xi_{1}}^{2}\right]
$$

By putting $s=1$ into Equation (13) we can get the first equation of quantum BBGKY as 


$$
i \hbar \frac{\partial}{\partial t} \hat{F}_{1}-\left[\hat{H}_{1}, \hat{F}_{1}\right]=\operatorname{Tr}_{s+1} \sum_{i=1}^{s}\left[\hat{v}_{12}, \hat{F}_{12}\right]
$$

Then we have the binary distribution function by substituting from Equation (19) into Equation (20):

$$
F_{12}^{q u}\left(\xi_{1}, P_{\xi_{1}}, \xi_{2}, P_{\xi_{2}}\right)=F_{1}^{q u}\left(\xi_{1}, P_{\xi_{1}}\right) F_{2}^{q u}\left(\xi_{2}, P_{\xi_{2}}\right)+g\left(\xi_{1}, \xi_{2}\right)
$$

where $g\left(\xi_{1}, \xi_{2}\right)$ is the correlation function.

Let us now study the model of dusty three component plasma i.e. the neutral system of point-like particles of positive and negative charges such as electrons, positrons and dust particles like ions. This model is an important model in both laboratory physics and space physics and has many applications [24]. By finding the quantum distribution functions of this model we can get the important thermodynamic functions such as the internal energy and the equation of state. Dust particles are heavier and slower in their velocities than electrons and positrons where for dusty plasma the relation between electrons, positrons, and dust (ion) density at equilibrium is $n_{e}=Z_{d} n_{i}+n_{p}$, where $n_{s}$ is the number density of $s^{\text {th }}$ species and $Z_{d}$ is the charge state of dust [24].

Substituting Equations (19) and (21) into (13) for $s=1,2$ we obtain

$$
\begin{aligned}
F_{12}^{q u}= & \frac{N^{2}}{8 V^{2}}\left(\frac{\beta}{\pi}\right)^{3}\left[\frac{\exp \left[-\beta \frac{P_{\xi_{e}}^{2}}{m_{e}}\right]}{m_{e}^{3}}+2 \frac{\exp \left[\frac{P_{\xi_{e}}^{2}}{2 m_{e}}+\frac{P_{\xi_{i}}^{2}}{2 m_{i}}\right]}{\left(m_{e} m_{i}\right)^{3 / 2}}\right] \\
& -\frac{e_{1} e_{2}}{K T \xi_{12}} \mathrm{e}^{-\kappa \xi_{12}}-\frac{e_{1} e_{2} \boldsymbol{p}_{\xi^{1}} \cdot \boldsymbol{p}_{\xi^{2}} \mathrm{e}^{-\kappa \xi_{12}}}{(m c)^{2} K T \xi_{12}}+\sum_{s=1}^{3} \frac{q_{s}^{2}}{m_{s}} \frac{1}{\xi_{s}}+\cdots
\end{aligned}
$$

\section{The Triplet Distribution Function}

The quantum triplet distribution function $F_{123}^{q u}$ defined in such a way that $F_{123}^{q u}\left(\xi_{1}, P_{\xi_{1}}, \xi_{2}, P_{\xi_{2}}, \xi_{3}, P_{\xi_{3}}, t\right) \mathrm{d} \xi_{1} \mathrm{~d} \xi_{2} \mathrm{~d} \xi_{3} \mathrm{~d} P_{\xi_{1}} \mathrm{~d} P_{\xi_{2}} \mathrm{~d} P_{\xi_{3}}$ is the probability of finding a particle of the type $1^{\text {th }}$ in the volume element $\mathrm{d} \xi_{1}$ surrounding $\xi_{1}$, with momentum in range $P_{\xi_{1}} \rightarrow P_{\xi_{1}}+\mathrm{d} P_{\xi_{1}}$, a particle of type $2^{\text {th }}$ in the volume element $\mathrm{d} \xi_{2}$ surrounding $\xi_{2}$, with momentum in range $P_{\xi_{2}} \rightarrow P_{\xi_{2}}+\mathrm{d} P_{\xi_{2}}$ and a particle of the type $3^{\text {th }}$ in the volume element $\mathrm{d} \xi_{3}$ surrounding $\xi_{3}$, with momentum in range $P_{\xi_{3}} \rightarrow P_{\xi_{3}}+\mathrm{d} P_{\xi_{3}}$ respectively at time $t$.

The quantum triplet distribution function $F_{123}^{q u}\left(\xi_{1}, P_{\xi_{1}}, \xi_{2}, P_{\xi_{2}}, \xi_{3}, P_{\xi_{3}}\right)$ is defined by the calculation of the interaction between three charged particles seems rather involved, because the force on particle 1 at time t would depend on the position and momentum of particles 2 and 3 at a retarded time. For simplification the quantum triplet distribution function $F_{123}^{q u}$ can be written as

$$
\begin{aligned}
F_{123}^{q u}(123)= & F_{1}^{q u}(1) F_{1}^{q u}(2) F_{1}^{q u}(3) \\
& +F_{1}^{q u}(1) g(23)+F_{1}^{q u}(2) g(13) \\
& +F_{1}^{q u}(3) g(12)+h(123)
\end{aligned}
$$


where $h(123)$ is the correlation function between particles 1, 2 and 3.

Substituting Equations (19) and (22) into (13) for $s=1,2,3$ we obtain

$$
\begin{aligned}
F_{123}^{q u}(123)= & \frac{N^{3}}{16 \sqrt{2} V^{3}}\left(\frac{\beta}{\pi}\right)^{\frac{9}{2}}\left[\frac{\exp \left[\frac{P_{\xi_{1}}^{2}}{2 m_{1}}+\frac{P_{\xi_{2}}^{2}}{2 m_{2}}+\frac{P_{\xi_{3}}^{2}}{2 m_{3}}\right]}{\left(m_{1} m_{2} m_{3}\right)^{3 / 2}}\right] \\
& +\frac{N}{V}\left(\frac{\beta}{2 \pi m}\right)^{3 / 2}\left\{\exp \left[\frac{-\beta}{2 m} P_{\xi_{1}}^{2}\right] g(23)+\exp \left[\frac{-\beta}{2 m} P_{\xi_{2}}^{2}\right] g(13)\right. \\
& \left.+\exp \left[\frac{-\beta}{2 m} P_{\xi_{3}}^{2}\right] g(12)\right\}+h(123)
\end{aligned}
$$

For three component plasma, we can use the two particle correlation function $g(12)$ which is given by

$$
g(12)=g_{1}\left(\xi_{12}\right)\left[1+\frac{\boldsymbol{p}_{\xi^{1}} \cdot \boldsymbol{p}_{\xi^{2}}}{(m c)^{2}}+\frac{\left(\boldsymbol{\xi}_{12} \cdot \boldsymbol{p}_{\xi^{1}}\right)\left(\boldsymbol{\xi}_{12} \cdot \boldsymbol{p}_{\xi^{2}}\right)}{2(m c)^{2} \xi_{12}^{2}}\right]
$$

where

$$
g_{1}\left(\xi_{12}\right)=-\frac{e_{1} e_{2}}{K T \xi_{12}} \mathrm{e}^{-\kappa \xi_{12}}
$$

$g_{1}\left(r_{12}\right)$ is the Debye-Hückel solution and the three particle correlation function $G(1,2,3)$ which is given by

$$
\begin{aligned}
h(123)= & g_{1}\left(\xi_{12}\right) g_{1}\left(\xi_{13}\right) g_{1}\left(\xi_{23}\right)\left[1+\frac{\boldsymbol{p}_{\xi^{1}} \cdot \boldsymbol{p}_{\xi^{2}}}{(m c)^{2}}+\frac{\boldsymbol{p}_{\xi^{1}} \cdot \boldsymbol{p}_{\xi^{3}}}{(m c)^{2}}\right. \\
& \left.+\frac{\boldsymbol{p}_{\xi^{2}} \cdot \boldsymbol{p}_{\xi^{3}}}{(m c)^{2}}+\sum_{i, j=1, i \neq j}^{3} \frac{\left(\boldsymbol{\xi}_{i j} \cdot \boldsymbol{p}_{\xi^{i}}\right)\left(\boldsymbol{\xi}_{i j} \cdot \boldsymbol{p}_{\xi^{j}}\right)}{2(m c)^{2} \xi_{i j}^{2}}+\cdots\right]
\end{aligned}
$$

Whatever particles 1, 2, 3 are, we can write the quantum triplet distribution function $F_{123}^{q u}(123)$ as

$$
\begin{aligned}
& F_{123}^{q u}(123)=\frac{N^{3}}{16 \sqrt{2} V^{3}}\left(\frac{\beta}{\pi}\right)^{\frac{9}{2}}\left[\frac{\exp \left[\frac{P_{\xi_{1}}^{2}}{2 m_{1}}+\frac{P_{\xi_{2}}^{2}}{2 m_{2}}+\frac{P_{\xi_{3}}^{2}}{2 m_{3}}\right]}{\left(m_{1} m_{2} m_{3}\right)^{3 / 2}}\right] \\
& +\frac{N}{V}\left(\frac{\beta}{2 \pi m}\right)^{3 / 2}\left\{\exp \left[\frac{-\beta}{2 m} P_{\xi_{1}}^{2}\right]\left[g_{1}\left(\xi_{23}\right)\left[1+\frac{\boldsymbol{p}_{\xi^{2}} \cdot \boldsymbol{p}_{\xi^{3}}}{(m c)^{2}}+\frac{\left(\boldsymbol{\xi}_{23} \cdot \boldsymbol{p}_{\xi^{2}}\right)\left(\boldsymbol{\xi}_{23} \cdot \boldsymbol{p}_{\xi^{3}}\right)}{2(m c)^{2} \xi_{23}^{2}}\right]\right)\right. \\
& +\exp \left[\frac{-\beta}{2 m} P_{\xi_{2}}^{2}\right]\left(g_{1}\left(\xi_{13}\right)\left[1+\frac{\boldsymbol{p}_{\xi^{1}} \cdot \boldsymbol{p}_{\xi^{3}}}{(m c)^{2}}+\frac{\left(\boldsymbol{\xi}_{13} \cdot \boldsymbol{p}_{\xi^{1}}\right)\left(\boldsymbol{\xi}_{13} \cdot \boldsymbol{p}_{\xi^{3}}\right)}{2(m c)^{2} \xi_{13}^{2}}\right]\right)
\end{aligned}
$$




$$
\begin{aligned}
& \left.+\exp \left[\frac{-\beta}{2 m} P_{\xi_{3}}^{2}\right]\left(g_{1}\left(\xi_{12}\right)\left[1+\frac{\boldsymbol{p}_{\xi^{1}} \cdot \boldsymbol{p}_{\xi^{2}}}{(m c)^{2}}+\frac{\left(\boldsymbol{\xi}_{12} \cdot \boldsymbol{p}_{\xi^{1}}\right)\left(\boldsymbol{\xi}_{12} \cdot \boldsymbol{p}_{\xi^{2}}\right)}{2(m c)^{2} \xi_{12}^{2}}\right]\right)\right\} \\
& +g_{1}\left(\xi_{12}\right) g_{1}\left(\xi_{13}\right) g_{1}\left(\xi_{23}\right)\left[1+\frac{\boldsymbol{p}_{\xi^{1}} \cdot \boldsymbol{p}_{\xi^{2}}}{(m c)^{2}}+\frac{\boldsymbol{p}_{\xi^{1}} \cdot \boldsymbol{p}_{\xi^{3}}}{(m c)^{2}}+\frac{\boldsymbol{p}_{\xi^{2}} \cdot \boldsymbol{p}_{\xi^{3}}}{(m c)^{2}}\right. \\
& \left.+\sum_{i, j=1, i \neq j}^{3} \frac{\left(\boldsymbol{\xi}_{i j} \cdot \boldsymbol{p}_{\xi^{i}}\right)\left(\boldsymbol{\xi}_{i j} \cdot \boldsymbol{p}_{\xi^{j}}\right)}{2(m c)^{2} \xi_{i j}^{2}}\right]
\end{aligned}
$$

\section{Quantum Distribution Functions of Saturn's Rings}

An alternative statistical description of planetary rings was formulated in a series of papers by Hämeen-Anttila [32] [33]. It uses a kinetic equation of Boltzmann type for the description of the evolution of the one-particle phase space distribution function, in a similar manner as gas-kinetics. Hämeen-Anttila gives analytical solutions for the collision integrals, where necessary in terms of appropriate approximations. The effect of self-gravity is taken into account in a self consistent manner in the local vertical gravity field of the disk and in its effect on close particle encounters. In principle the theory can treat the average effect of particle surface irregularities stochastically and it is formulated so that it can be extended to describe particle fragmentation and coagulation.

The biggest advantage is that the balance equations for mass, stress, and scale hight of the ring, are given analytically as partial differential equations. Thus, the theory can be applied to investigate the dynamical evolution of a planetary ring. The disk's self-gravity potential $\phi_{\text {disk }}$ couples to the surface mass density through Poisson's equation [34]

$$
\nabla^{2} \phi_{d i s k}=4 \pi a \sigma \delta
$$

where $\sigma$ is the surface mass density, $a$ is gravitation parameter and $\delta$ is the Dirac delta function.

$$
\frac{1}{h_{1} h_{2} h_{3}}\left[\frac{\partial}{\partial \xi_{1}}\left(\frac{h_{2} h_{3}}{h_{1}} \frac{\partial \phi_{\text {disk }}}{\partial \xi_{1}}\right)+\frac{\partial}{\partial \xi_{2}}\left(\frac{h_{1} h_{3}}{h_{2}} \frac{\partial \phi_{\text {disk }}}{\partial \xi_{2}}\right)+\frac{\partial}{\partial \xi_{3}}\left(\frac{h_{2} h_{1}}{h_{3}} \frac{\partial \phi_{\text {disk }}}{\partial \xi_{3}}\right)\right]=4 \pi a \sigma \delta
$$

where $h_{1}, h_{2} h_{3}$ is the natural basis vectors. The Kinetic theory describes the evolution of the local velocity distribution function of an ensemble of particles in terms of the Boltzmann equation or a suitable generalization of it, like Enskog's theory of hard sphere gases. The kinetic equation can be derived from Liouville's theorem, appearing as the leading equation in a hierarchy of equations describing $\mathrm{n}$-particle distribution functions in phase space and neglecting correlations between particle pairs. Then the one particle distribution function in Equation (19) in in the local vertical gravity field can be given by

$$
F_{1}^{q u}\left(\xi_{1}, P_{\xi_{1}}\right)=\frac{N}{V}\left(\frac{\beta}{2 \pi m}\right)^{3 / 2} \exp \left[\frac{-\beta}{2 m} P_{\xi_{1}}^{2}-e \phi\right]
$$


Kinetic theory allows us to incorporate the full complexity of the dynamics of a planetary ring in a statistical description, such as the effects of the motion of ring particles on curved orbits between inelastic collisions, their finite size, the anisotropy of the velocity dispersion, and in principle also coagulation and fragmentation of the ring particles.

The collision motion of an ensemble of identical particles in the plane, in the frame of reference rotating with angular velocity $\omega$ can be described by the Boltzmann equation [35] in Curvilinear Coordinates:

$$
\begin{aligned}
& \frac{\partial f}{\partial t}+\frac{p_{\xi_{1}}}{m h_{1}} \frac{\partial f}{\partial \xi_{1}}+\left(\omega+\frac{p_{\xi_{2}}}{m h_{2}}\right) \frac{\partial f}{\partial \xi_{2}}+\left(\frac{2 \omega}{m} p_{\xi_{2}}+\frac{p_{\xi_{2}}^{2}}{m h_{2}}-\frac{\partial \phi_{1}}{h_{1} \partial \xi_{1}}\right) \frac{\partial f}{\partial p_{\xi_{1}}} \\
& =\left(\frac{\kappa^{2}}{2 m h_{1} \omega} p_{\xi_{1}}+\frac{p_{\xi_{1}} p_{\xi_{2}}}{m^{2} h_{1} h_{2}}+\frac{\partial \phi_{1}}{h_{2} \partial \xi_{2}}\right) \frac{\partial f}{\partial p_{\xi_{2}}}+\left(\frac{\partial f}{\partial t}\right)_{c l}
\end{aligned}
$$

where $\xi_{1}, \xi_{2}, \xi_{3}$ was defined by Equation (1) and $\left(\frac{\partial f}{\partial t}\right)_{c l}$ is the collision integral which takes into account effects due to the discrete-point nature of the gravitational charges, or collision effects (including diffusion in space and velocity), and defines the change of the distribution function $f(r, v, t)$ arising from ordinary interparticle collisions (in a plasma this term represents the change of $f$ arising from collisions with particles at distances shorter than a Debye length). The Boltzmann form for the collision integral is based on an assumption that the duration of a collision is much less than the time between collisions instantaneous collisions are considered [36]. The simple Krook integral in the case of a two-dimensional disk of identical particles has the form

$$
\left(\frac{\partial f}{\partial t}\right)_{c l}=-v_{c}\left(f-f_{0}\right)
$$

where $f$ is the actual distribution function of particles and $f_{0}$ is the steady-state equilibrium distribution function (Shu and Stewart, 1985) [35]. The equilibrium axially symmetric distribution function is the Maxwellian with the surface density $\sigma_{0}$ :

$$
f_{0}=\frac{\sigma_{0}}{2 \pi c^{2}} \exp \left(-\frac{p_{\xi}^{2}}{2 m^{2} c^{2}}\right)
$$

The gravitational potential of a rotating oblate planet

$$
\phi(\xi, \beta)=-\frac{a M_{0}}{2}\left[1+\left(\frac{R}{\xi}\right)^{2}\left(\frac{1}{2}-\frac{3}{2} \sin ^{2} \beta\right) I_{2}\right]
$$

where $r$ is the distance from the center of the planet to the point at which the potential is sought, $\beta$ is the planetocentric latitude of the point, $\mathrm{R}$ is the radius of the planet, and $M_{0}$ is its mass. For saturn $I_{2}=0.017, M_{0}=5.7 \times 10^{29} \mathrm{~g}$ and $R=6 \times 10^{7} \mathrm{~m}$ for more exact values of the parameters see Ref. [37].

\section{Conclusions}

In this work we obtained the quantum binary and triplet distribution functions 
of dusty plasma; the calculation is based on curvilinear coordinates and the Bogoliubov-Born-Green-Kirkwood-Yvon (BBGKY) hierarchy. We consider only the thermal equilibrium plasma. The model under consideration is the three-component dusty plasma i.e. neutral system of point like particles of positive and negative charges (electrons and positrons) interspersed with dust particles (ions) [24]. Figure 1 and Figure 2 show the effect of dust on the quantum binary and triplet distribution functions. From these figures we note that the existence of dust makes a fluctuation in binary and triplet distribution functions in the range of $\xi=(0.5,2.5)$. It was shown that the local stability criterion obtained from the computer models is in general agreement with the theoretical prediction as outlined in the present paper.

The first derivation of reactive quantum Boltzmann equations by Olmstead and Curtiss [18] starts with the Wigner transformed version of the standard BBGKY hierarchy for a system of stable atomic and diatomic constituents. Also, they calculated the quantum triplet distribution function of moderately dense gases. Alavi et al. [19] described the time evolution of a one-particle statistical distribution or density operator, influenced by the interaction of another particle which represents the effect of all other particles in the system.

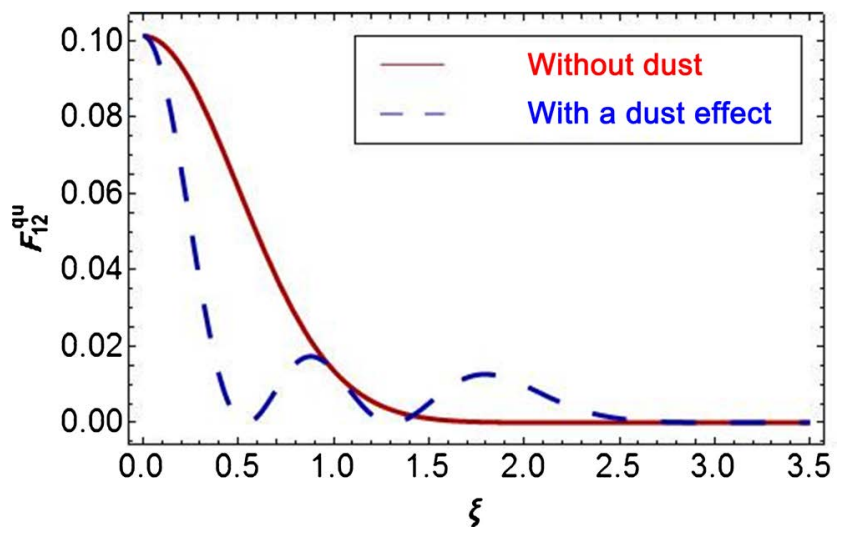

Figure 1. The quantum binary distribution function from Equation (22) without dust effect (solid line) and with a dust effect (dashed line).

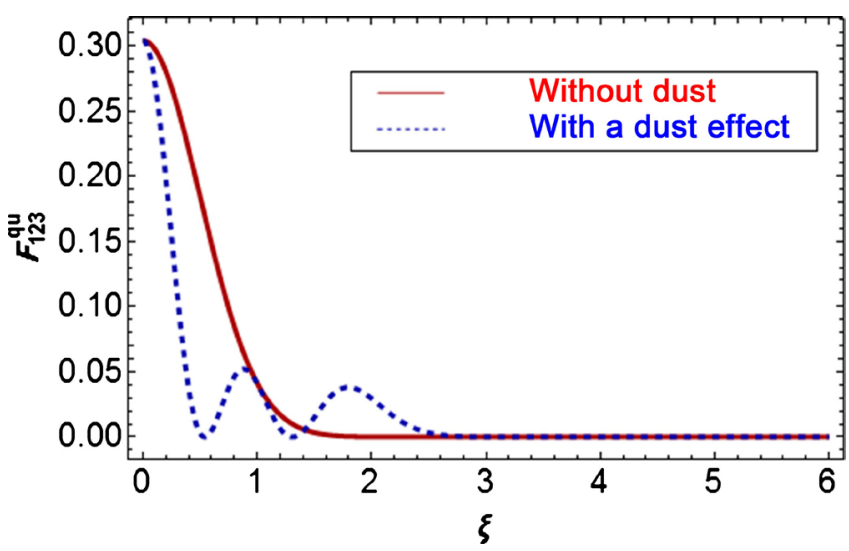

Figure 2. The quantum triplet distribution function from Equation (28) without dust effect (solid line) and with a dust effect (dashed line). 
Figure 3 and Figure 4 show the comparison between the quantum binary and triplet distribution function based on our result without dust effect (solid line), Alavi et al. [19] (dashed line) and from Olmstead and Curtiss [18] (dot-dashed line). We note from this comparison the convergence between the results obtained and the results of the references [19] and [18].

Figure 5 and Figure 6 show the comparison between the quantum binary and triplet distribution function based on our result with the effect of dust, Alavi et al. [19] and from Olmstead and Curtiss [18]. After taking into account the effect of dust, we noticed that our results are closer to the results obtained by Olmstead and Curtiss [18] in the range of $\xi=(0,0.5)$, but with the increase in the value of the variable $\xi$ in the range of $\xi=(0.5,1)$ the results are closer to results obtained by Alavi et al. [19].

Initial quantum curvilinear coordinates phase-space $\left(\xi, V_{\xi}\right)$ for dusty plasma electrons (gray color), positrons (red color) and dust (orange color) was given in Figure 7. Figure 8 shows the quantum curvilinear coordinates phase-space $\left(\xi, V_{\xi}\right)$ for dusty plasma based on Particle-In-Cell (PIC) simulation methods.

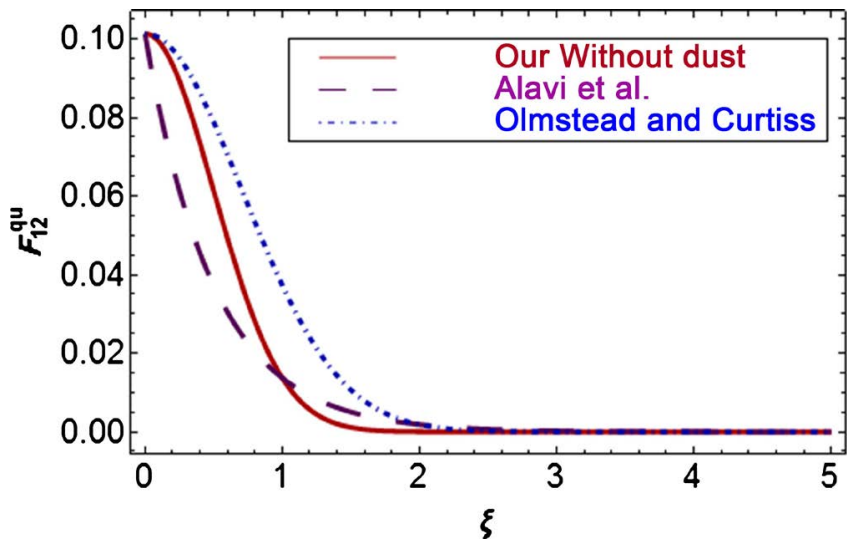

Figure 3. The comparison between the quantum binary distribution function based on our result without dust effect (solid line), Alavi et al. [19] (dashed line) and from Olmstead and Curtiss [18] (dot-dashed line).

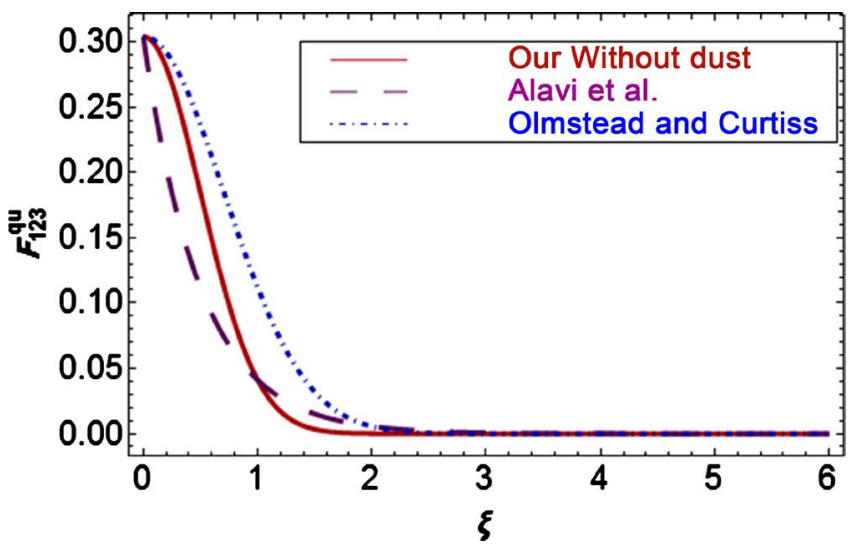

Figure 4. The comparison between the quantum triplet distribution function based on our result without dust effect (solid line), Alavi et al. [19] (dashed line) and from Olmstead and Curtiss [18] (dot-dashed line). 


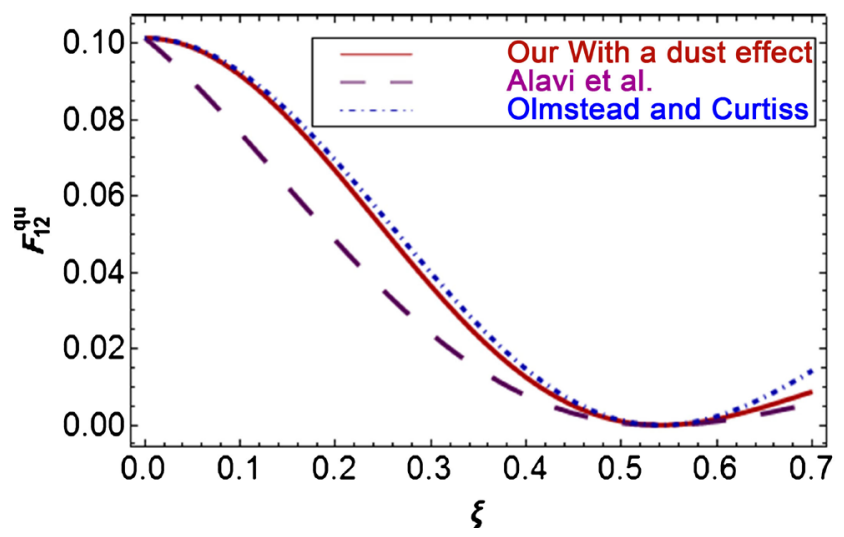

Figure 5. The comparison between the quantum binary distribution function based on our result with a dust effect (solid line), Alavi et al. [19] (dashed line) and from Olmstead and Curtiss [18] (dot-dashed line).

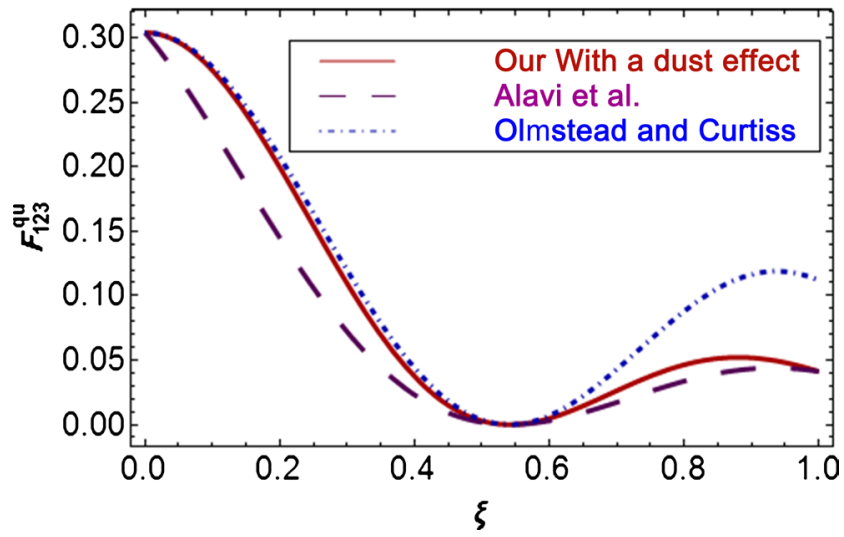

Figure 6. The comparison between the quantum triplet distribution function based on our result without dust effect (solid line), Alavi et al. [19] (dashed line) and from Olmstead and Curtiss [18] (dot-dashed line).

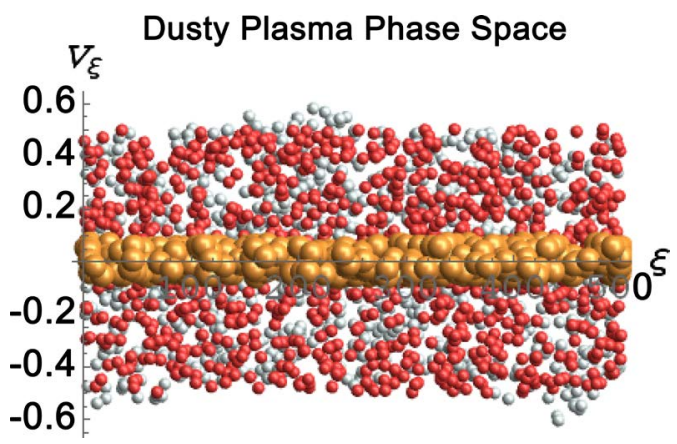

Figure 7. Initial quantum curvilinear coordinates phase-space $\left(\xi, V_{\xi}\right)$ for dusty plasma electrons (gray color), positrons (red color) and dust (orange color).

We observe from the phase space that the speed of dust particles is much lower than the speed of electrons and positrons. The density of dust particles varies due to the large volume of dust particles.

The quantum binary distribution function for dusty plasma was given in Figure 9. The quantum triplet distribution function for dusty plasma see Figure 10 
with $\xi_{1}=\xi_{2} \neq \xi_{3}$.

Figure 11 and Figure 12 show the quantum binary and triplet distribution functions for dusty plasma in curvilinear phase space $\left(\xi, P_{\xi}\right)$. These solutions are important in different branches of physics and other areas of applied sciences and can provide help for researchers to study and understand the physical interpretation of the dusty plasma model.

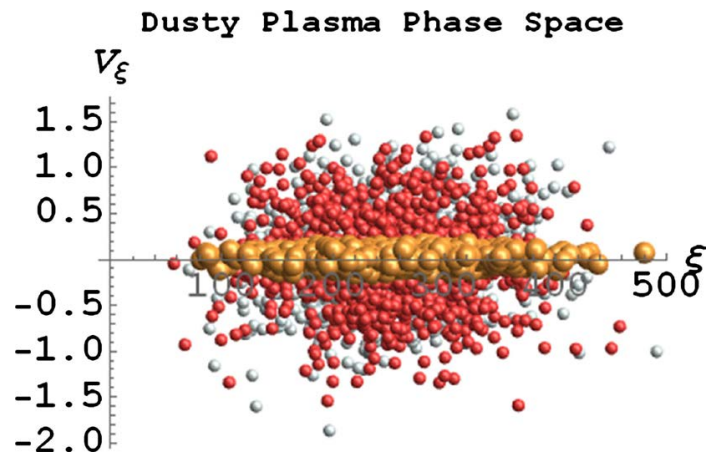

Figure 8. The quantum curvilinear coordinates phase-space $\left(\xi, V_{\xi}\right)$ for dusty plasma electrons (gray color), positrons (red color) and dust (orange color) based on Particle-In-Cell (PIC) simulation methods.

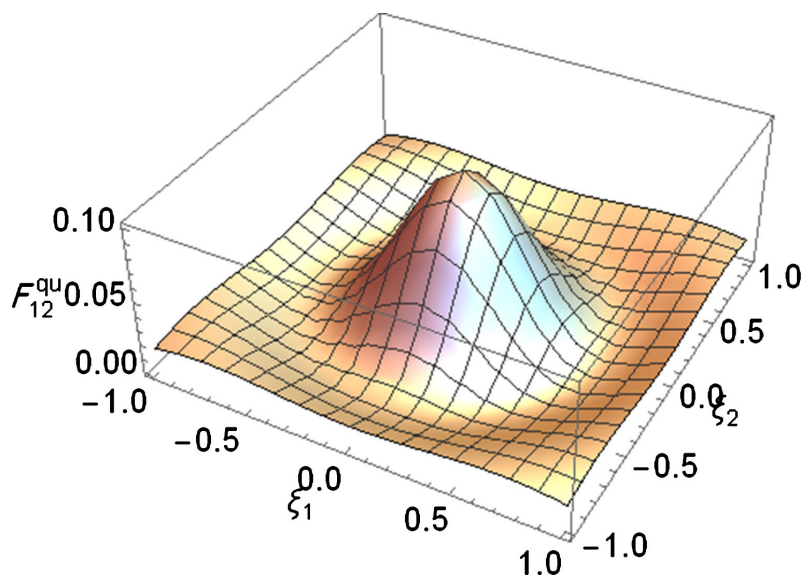

Figure 9. The quantum binary distribution function for dusty plasma with $\xi_{1} \neq \xi_{2}$.

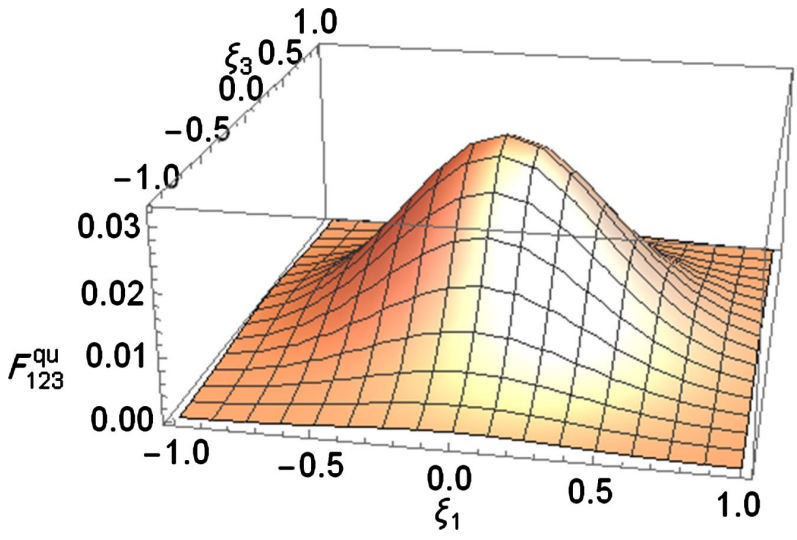

Figure 10. The quantum triplet distribution function for dusty plasma with $\xi_{1}=\xi_{2} \neq \xi_{3}$. 


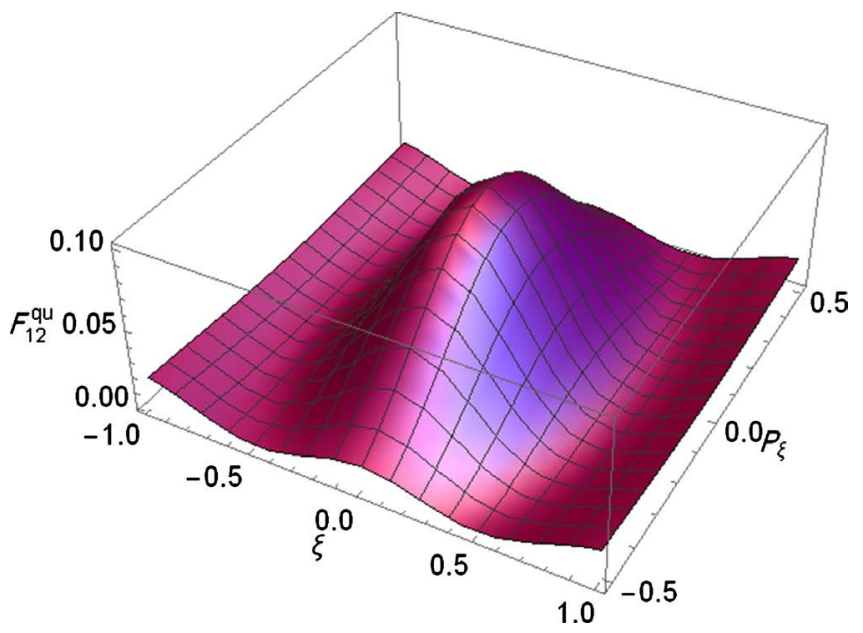

Figure 11. The quantum binary distribution function for dusty plasma in curvilinear phase-space $\left(\xi, P_{\xi}\right)$.

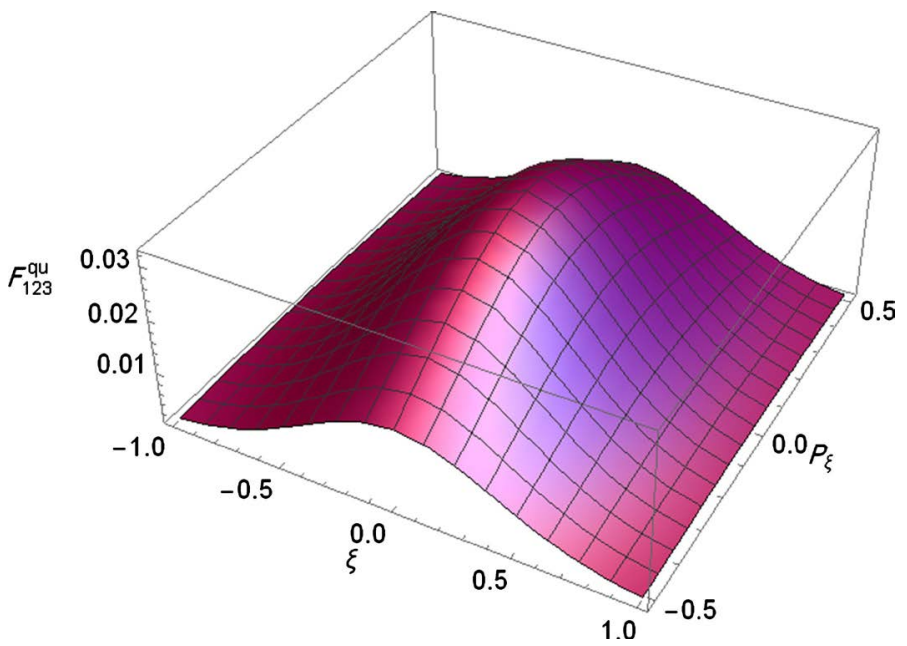

Figure 12. The quantum triplet distribution function for dusty plasma in curvilinear phase-space $\left(\xi, P_{\xi}\right)$.

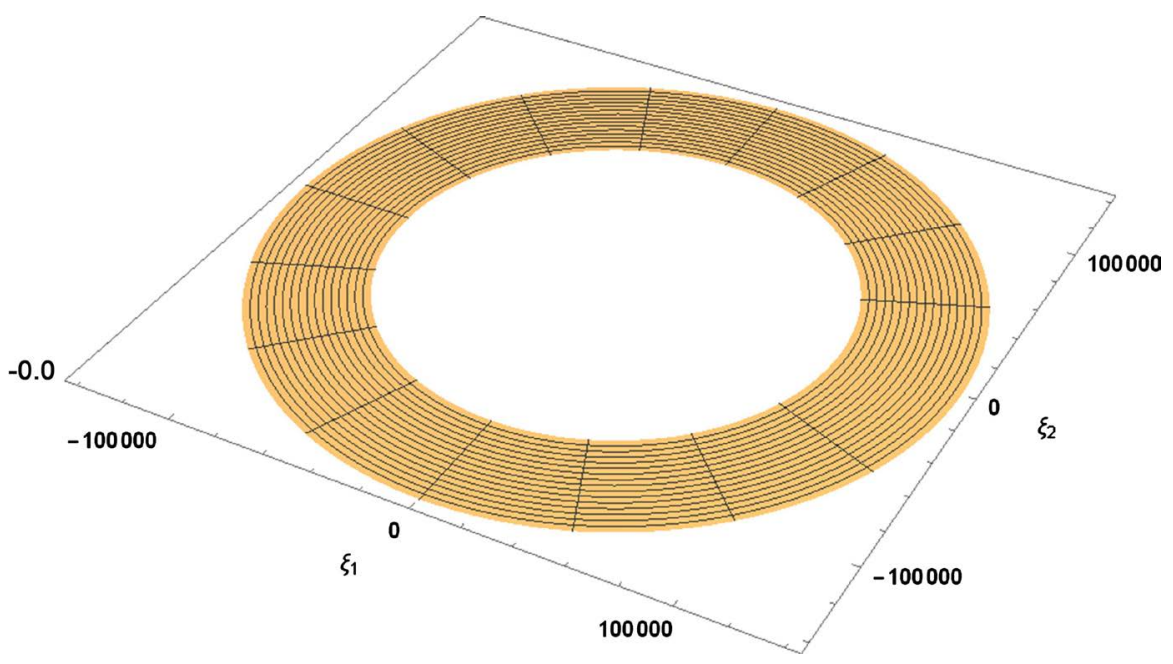

Figure 13. Model of Saturn planet rings in the radius interval $(92,000 \mathrm{~km} ; 139,350 \mathrm{~km})$. 


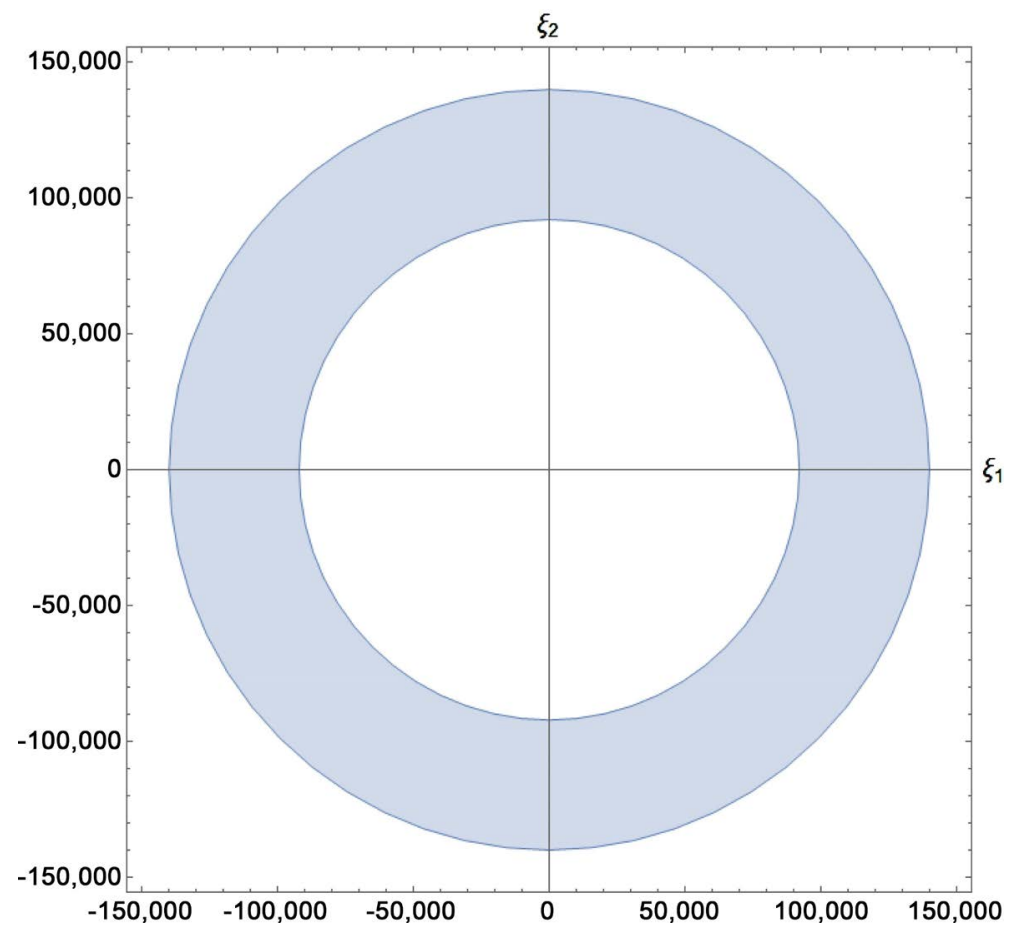

Figure 14. 2D Model of Saturn planet rings in the radius interval $(92,000 \mathrm{~km} ; 139,350 \mathrm{~km})$.

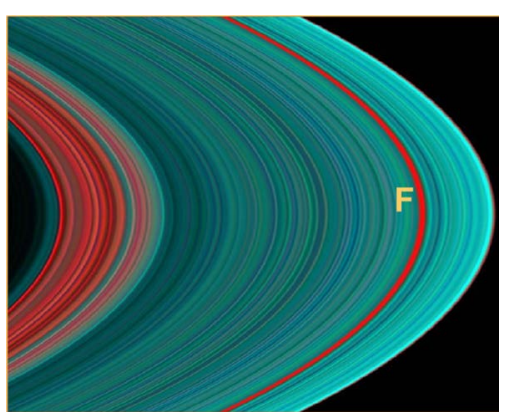

Figure 15. Ultraviolet ring's image; red color refer to dust and blue for water ice.

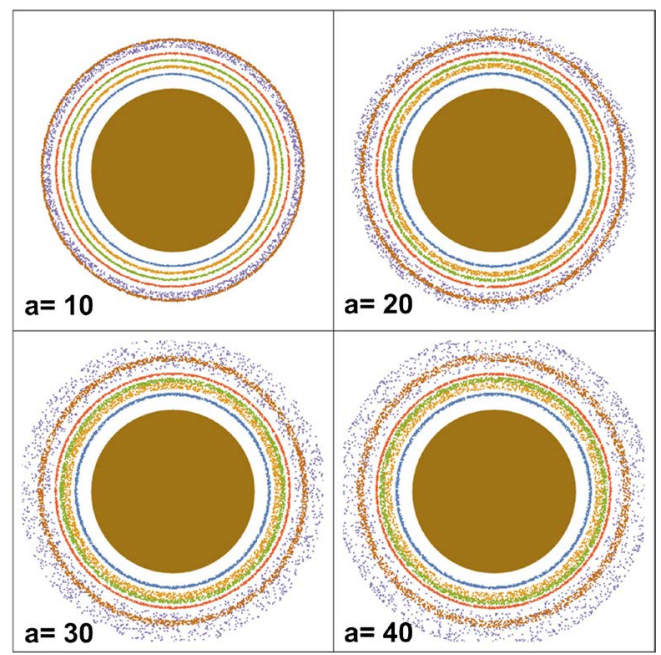

Figure 16. Simulation of dusty plasma for sturan rings for different values of gravitation parameter in the radius interval $(92,000 \mathrm{~km} ; 139,350 \mathrm{~km})$. 
Model of Saturn planet rings in the radius interval $(92,000 \mathrm{~km}, 139,350 \mathrm{~km})$ was given in Figure 13 and Figure 14. Figure 15 shows ultraviolet ring's image; red color refers to dust and blue for water ice. Figure 16 shows the Simulation model of dusty plasma for Saturn rings for different values of gravitation parameter. Also, we note from this figure when gravitation parameter increased the dispersion of the presence of particles becomes more visible than the lower values of the gravitation parameter.

\section{Conflicts of Interest}

The authors declare no conflicts of interest regarding the publication of this paper.

\section{References}

[1] Hussein, N.A. and Hassan, A.R. (1990) Quantum Binary Distribution of High Temperature Plasma. Bulletin of the Faculty of Science. Assiut University, 19, 39-98.

[2] Kraeft, W.D., Vorberger, J., Gericke, D.O. and Schlanges, M. (2007) Thermodynamic Functions for Plasmas beyond Montroll Ward. Contributions to Plasma Physics, 47, 253-261. https://doi.org/10.1002/ctpp.200710034

[3] Bogoliubov, N.N. (1962) Probleme der Dynamischen Theorie in der Statistischen Physik. Studies in Statistical Mechanics, 10, 275.

[4] Kraeft, W.D., Kremp, D., Ebeling, W. and Röpke, G. (1986) Quantum Statistics of Charged Particle Systems. Akademie Verlag, Berlin. https://doi.org/10.1007/978-1-4613-2159-0

[5] Kaniadakis, G. (2003) BBGKY Hierarchy Underlying Many-Particle Quantum Mechanics. Physics Letters A, 310, 377-382. https://doi.org/10.1016/S0375-9601(03)00448-1

[6] Fesciyan, S. (1973) The BBGKY Hierarchy in Quantum Statistical Mechanics. Communications in Mathematical Physics, 30, 11-22. https://doi.org/10.1007/BF01646684

[7] Hussein, N.A., Osman, A.-N.A., Eisa, D.A. and Abbas, R.A. (2014) Quantum Binary and Triplet Distribution Functions of Plasma by using Green's Function. Contributions to Plasma Physics, 54, 815-826. https://doi.org/10.1002/ctpp.201400016

[8] Barker, A. (1968) A Quantum Mechanical Calculation of the Radial Distribution Function for a Plasma. Australian Journal of Physics, 21, 121-128. https://doi.org/10.1071/PH680121

[9] Satoh, H., Tanaka, M. and Katsura, S. (1984) Triplet Distribution Function of the Fluid with the Square Well Potential. Molecular Physics, 53, 1195-1208. https://doi.org/10.1080/00268978400102941

[10] Lado, F. (1991) Integrals over the Triplet Distribution Function without the Triplet Distribution Function. Molecular Physics, 72, 1387-1395. https://doi.org/10.1080/00268979100100971

[11] Taylor, M.P. and Lipson, J.E.G. (1992) On the Born-Green-Yvon Equation and Triplet Distributions for Hard Spheres. The Journal of Chemical Physics, 97, 4301. https://doi.org/10.1063/1.463932

[12] Zahn, K., Maret, G., Rub, C. and Grunberg, H. (2003) Three-Particle Correlations in 
Simple Liquids. Physical Review Letters, 91, Article ID: 115502. https://doi.org/10.1103/PhysRevLett.91.115502

[13] Russ, C., Brunner, M., Bechinger, C. and Grunberg, H. (2005) Three-Body Forces at Work: Three-Body Potentials Derived from Triplet Correlations in Colloidal Suspensions. Europhysics Letters, 69, 468. https://doi.org/10.1209/epl/i2004-10345-8

[14] Jorge, S., Lomba, E. and Abascal, J.L.F. (2002) Theory and Simulation of the Triplet Structure Factor and Triplet Direct Correlation Functions in Binary Mixtures. Chemical Physics, 116, 730. https://doi.org/10.1063/1.1426418

[15] Ludwig, P., Thomsen, H., Balzer, K., Filinov, A. and Bonitz, M. (2010) Tuning Correlations in Multi-Component Plasmas. Plasma Physics and Controlled Fusion, 52, Article ID: 124013. https://doi.org/10.1088/0741-3335/52/12/124013

[16] Hakim, R. (2011) Introduction to Relativistic Statistical Mechanics: Classical and Quantum. World Scientific, USA. https://doi.org/10.1142/7881

[17] Hansen, J.P. (1973) Statistical Mechanics of Dense Ionized Matter. I. Equilibrium Properties of the Classical One-Component Plasma. Physical Review A, 8, 3096. https://doi.org/10.1103/PhysRevA.8.3096

[18] Olmstead, R.D. and Curtiss, C.F. (1975) A Quantum Kinetic Theory of Moderately Dense Gases. III. The Effect of Bound States on the Transport Coefficients. The Journal of Chemical Physics, 63, 1966. https://doi.org/10.1063/1.431531

[19] Alavi, S., Wei, G.W. and Snider, R.F. (1998) Chain Relations of Reduced Distribution Functions and Their Associated Correlation Functions. The Journal of Chemical Physics, 108, 706. https://doi.org/10.1063/1.475430

[20] Błaszak, M. and Domański, Z. (2013) Canonical Quantization of Classical Mechanics in Curvilinear Coordinates. Invariant Quantization Procedure. Annals of Physics, 339, 89-108. https://doi.org/10.1016/j.aop.2013.08.014

[21] Kjaergaard, H.G. and Mortensen, O.S. (1990) The Quantum Mechanical Hamiltonian in Curvilinear Coordinates: A Simple Derivation. American Journal of Physics, 58, 344-347. https://doi.org/10.1119/1.16167

[22] Fichtl, C., Finn, J. and Cartwright, K. (2012) An Arbitrary Curvilinear-Coordinate Method for Particle-in-Cell Modeling. Computational Science \& Discovery, 5, Article ID: 014011. https://doi.org/10.1088/1749-4699/5/1/014011

[23] Mendis, D.A. (1997) Advances in Dusty Plasmas: Proceedings of the International Conference on the Physics of Dusty Plasmas. In: Shukla, P.K., Mendis, D. and Desai, T., Eds., Advances in Dusty Plasmas, World Scientific, Singapore, 3-19.

[24] Shukla, P.K. and Mamun, A.A. (2002) Introduction to Dusty Plasma Physics. IOP, London, UK.

[25] Ferdousi, M., Yasmin, S., Ashraf, S. and Mamun, A.A. (2015) Ion-Acoustic Shock Waves in Nonextensive Electron-Positron-Ion Plasma. Chinese Physics Letters, 32, Article ID: 015201. https://doi.org/10.1088/0256-307X/32/1/015201

[26] Wang, Y. and Zhang, J. (2008) Soliton and Chaotic Structures of Dust Ion-Acoustic Waves in Quantum Dusty Plasmas. Physics Letters A, 372, 6509-6517. https://doi.org/10.1016/j.physleta.2008.09.004

[27] Goertz, C. (1989) Dusty Plasmas in the Solar System. Reviews of Geophysics, 27, 271-292. https://doi.org/10.1029/RG027i002p00271

[28] Dougherty, M., Esposito, L. and Krimigis, S. (2009) Saturn from Cassini-Huygens. Springer Science \& Business Media, Berlin. https://doi.org/10.1007/978-1-4020-9217-6

[29] Smith, L., Soderblom, R., Batson, P., Bridges, J., Inge, H. and Bunker, A. (1982) A 
New Look at the Saturn System: The Voyager 2 Images. Science, 215, 504-537. https://doi.org/10.1126/science.215.4532.504

[30] Gyergyek, T. and Kovačič, J. (2014) Potential Formation in Front of an Electrode Close to the Plasma Potential Studied by PIC Simulation. Contributions to Plasma Physics, 54, 647-668. https://doi.org/10.1002/ctpp.201300036

[31] Reinmüller, K. (1998) Determination of the Plasma Potential Using Emissive Probes-Implications from PIC Simulations. Contributions to Plasma Physics, 38, 7-12. https://doi.org/10.1002/ctpp.19980380106

[32] Hämeen-Anttila, K.A. (1975) Statistical Mechanics of Keplerian Orbits. Astrophysics and Space Science, 37, 309-333. https://doi.org/10.1007/BF00640356

[33] Hämeen-Anttila, K.A. (1976) Statistical Mechanics of Keplerian Orbits. II-Dispersion in Particle Size. Astrophysics and Space Science, 43, 145-174. https://doi.org/10.1007/BF00640563

[34] Pollack, J.B. (1975) The Rings of Saturn. Space Science Reviews, 18, 3-93. https://doi.org/10.1007/BF00350197

[35] Shu, F.H. and Stewart, G.R. (1985) The Collisional Dynamics of Particulate Disks. Icarus, 62, 360-383. https://doi.org/10.1016/0019-1035(85)90181-2

[36] Griv, E., Gedalin, M., Eichler, D. and Yuan, C. (2000) A Gas-Kinetic Stability Analysis of Self-Gravitating and Collisional Particulate Disks with Application to Saturn's Rings. Planetary and Space Science, 48, 679-698. https://doi.org/10.1016/S0032-0633(00)00037-4

[37] https://solarsystem.nasa.gov/planets/saturn/overview/ 